\title{
SOLUTIONS OF FIRST ORDER DIFFERENTIAL EQUATIONS WHICH ARE SOLUTIONS OF LINEAR DIFFERENTIAL EQUATIONS OF HIGHER ORDER ${ }^{1}$
}

\section{LAWRENCE GOLDMAN}

Let $\mathcal{F}$ be an ordinary differential field (i.e., a field with a given derivation) of characteristic zero. An element $z$ belonging to a differential field extension of $\mathcal{F}$ is said to be of order $r$ over $\mathcal{F}$ if the lowest order irreducible differential equation with coefficients in $\mathcal{F}$ that $z$ satisfies is of order $r$. It follows that $z$ is of order $r$ over $\mathcal{F}$ if and only if the degree of transcendency of $\mathfrak{F}\langle z\rangle$ over $\mathcal{F}$ is $r$.

In [4] Ritt proved that if $P, Q \in F\{y\}$ and $Q$ vanishes for every zero of $P$ then the sum of the lowest (highest) degree terms of $Q$ vanishes for every zero of the sum of the lowest (highest) degree terms of $P$.

For our purpose we need the following slight generalization; it can be obtained either by essentially the same proof as used by Ritt, or as an almost immediate corollary of this theorem.

Theorem 1. Let $P, Q \in \mathfrak{F}\{y\}$ and let $Q$ vanish for every zero of $P$ which is of order $\geqq r$ over $\mathfrak{F}$; then the sum of lowest (highest) degree terms of $Q$ vanishes for every zero of the sum of lowest (highest) degree terms of $P$ which is of order $\geqq r$ over $\mathcal{F}$.

Theorem 2. Let $z$ be a zero of an nth order linear differential polynomial $L(y) \in \mathcal{F}\{y\}$ and let the order of $z$ over $\mathcal{F}$ be 1 . Then there exists an integer $r, 0 \leqq r<n$, such that $z^{(r)}$ is a zero of an irreducible first order differential polynomial $P(y) \in \mathcal{F}\{y\}$ the sum of whose highest degree terms is of order 1 and $\boldsymbol{z}^{(r)}$ is of order 1 over $\mathfrak{F}$.

Proof. Since $z$ is of order 1 over $F, F\langle z\rangle=F\left(z, z^{\prime}\right)$, which is an algebraic function field of one variable over $\mathcal{F}$. Let $v$ be an infinite valuation on $\mathcal{F}\left(z, z^{\prime}\right)$ such that $v$ is trivial on $\mathcal{F}$ and for any $Q(z), R(z)$ $\in \mathcal{F}[z] v(Q / R)=$ degree of $R$-degree of $Q$. Since $z$ is a zero of an $n$th order linear differential polynomial we can not have $v\left(z^{(s+1)}\right)<v\left(z^{(s)}\right)$ for all $s$ less than $n$. Let $r$ be the smallest integer such that $v\left(z^{(r+1)}\right)$ $\geqq v\left(z^{(r)}\right)$; then the order of $z^{(r)}$ over $\mathcal{F}$ is 1 . For, $z^{(r)} \in \mathcal{F}\langle z\rangle$ so that the order of $z^{(r)}$ over $\mathcal{F}$ is $\leqq 1$; if $z^{(r)}$ were algebraic over $\mathcal{F}$ then $v\left(z^{(r)}\right)$ would be zero, which is greater than $v(z)$, contradicting our assumption on the minimality of $r$. Let $P(y) \in \mathcal{F}\{y\}$ be the first order ir-

Received by the editors March 19, 1959.

1 This research was supported by the National Science Foundation. 
reducible differential polynomial which vanishes for $z^{(r)}$. Since at least two terms of $P\left(z^{(r)}\right)$ must have the same smallest value under the given valuation $v$, it follows that the highest degree terms of $P(y)$ are of order 1 .

A homogeneous linear differential polynomial $L(y) \in \mathcal{F}\{y\}$ is said to be linearly reducible over $\mathcal{F}$ if there exist homogeneous linear differential polynomials $M(y), N(y) \in F\{y\}$, each one of positive order, such that

$$
L(y)=M(N(y)) .
$$

If no such decomposition exists we say $L(y)$ is linearly irreducible over $\mathfrak{F}$.

Theorem 3. Let $L(y) \in \mathcal{F}\{y\}$ be a homogeneous linear differential polynomial linearly irreducible over $\mathcal{F}$. If a zero $z$ of $L(y)$ is of order 1 over $\mathcal{F}$, then there exists a fundamental system of zeros $\left(u_{1}, \cdots, u_{n}\right)$ of $L(y)$ such that $u_{i}^{\prime} / u_{i}, i=1, \cdots, n$, is algebraic over $\mathfrak{F}$.

Proof. Since $L(y)$ is linearly irreducible over $\mathcal{F}$ it suffices ${ }^{2}$ to show the existence of one zero $u$ of $L(y)$ such that $u^{\prime} / u$ is algebraic over $F$ [2]. By Theorem 2 there exists an integer $r$ such that $z^{(r)}$ is a zero of a first order differential polynomial $P(y) \in \mathcal{F}\{y\}$ the sum of whose highest degree terms is of order 1 . Because $L(y)$ is linearly irreducible there exist homogeneous linear differential polynomials $M(y)$, $N(y) \in \mathfrak{F}\{y\}, M(y)$ linearly irreducible over $\mathcal{F}$, such that $M\left(z^{(r)}\right)=0$ and, for any nontrivial zero $w$ of $M(y), N(w)$ is a nontrivial zero of $L(y)[5$, vol. 2, pp. 164, 165]. By Theorem 1 there exists a nontrivial zero $w$ of the sum of the highest degree terms of $P(y)$ such $M(w)=0$. Since $w$ is a zero of a homogeneous first order differential polynomial $w^{\prime} / w$ is algebraic over $\mathcal{F}$. Let $u=N(w)$ then $L(u)=0$. Since $w^{\prime} / w$ is algebraic over $\mathcal{F}, u=k w$, where $k$ belongs to an algebraic extension of $\mathcal{F}$, so that $u^{\prime} / u$ is algebraic over $\mathcal{F}$ and our theorem follows.

DEFINITION. If the lowest order linear differential polynomial which vanishes for $z$ is of order $n$, then we say that the linear order of $z$ over $\mathcal{F}$ is $n$. Let the linear order of $z$ over $\mathcal{F}$ be $n$ and let $V$ be the set of all linear differential polynomials in $z$ with coefficients in $\mathcal{F}$. $V$ is, in an obvious way, an $n+1$ dimensional vector space over $\mathcal{F}$ so that the linear order of any element of $V$ over $\mathcal{F}$ is $\leqq n$.

${ }^{2}$ Loewy assumes that $\mathcal{F}$ has an algebraically closed field of constants and uses in his proof the automorphisms of a Picard-Vessiot extension of $\mathcal{F}$. It is easily seen, however, that by the substitution of relative isomorphisms over $\mathcal{F}$ for his automorphisms, the proof remains valid. For, we can use the theorem by Kolchin [1] that an element $t$ belonging to $\mathcal{K}$, a differential field extension of $\mathcal{F}$, which is left invariant by every isomorphism of $\mathcal{K}$ over $\mathcal{F}$, belongs to $\mathcal{F}$. 
THEOREM 4. Let $z$ be a zero of a first order differential polynomial $P(y) \in \mathcal{F}\{y\}$ and of a linear differential polynomial $L(y) \in \mathcal{F}\{y\}$, and let $\overline{\mathcal{F}}$ be the algebraic closure of $\mathcal{F}$. There exists $u \in \bar{F}\langle z\rangle$ such that $z$ is algebraic over $\mathcal{F}\langle u\rangle$ and the linear order of $u$ over $\bar{F}$ is 1 .

REMARK. If a first order differential polynomial $P(y) \in \mathcal{F}\{y\}$ factors over $\bar{F}$ into linear factors, it is well known that any zero $u$ of $P(y)$ is a zero of a linear differential polynomial of higher order with coefficients in $\mathcal{F}[3]$. Also, any polynomial $z \in \bar{F}\{u\}$ is a zero of a first order differential polynomial $Q(y) \in \mathcal{F}\{y\}$ and of a linear differential polynomial with coefficients in $\mathcal{F}$. $Q(y)$ may remain irreducible over $\bar{F}$. For example, let $\mathcal{F}$ be the field of rational numbers, $u=e^{x}$, $z=e^{x}+e^{2 x} ; Q(y)=\left(2 y-y^{\prime}\right)^{2}-\left(y^{\prime}-y\right)$ is, obviously, irreducible over $\bar{F}$. Our theorem states that besides the obvious cases just mentioned there is only one more possibility for an element $z$ to be simultaneously a zero of a first order and of a linear differential polynomial; namely that $z$ belongs to an algebraic extension of $\bar{F}\langle u\rangle$ where $u$ is a zero of a first order linear differential polynomial with coefficients in $\overline{\mathfrak{F}}$.

Proof OF Theorem. If $z \in \bar{F}$ we take $u=z$. Let the order of $z$ over $\mathcal{F}$ (and hence over $\overline{\mathcal{F}}$ ) be 1 . Let $V$ be the vector space of all linear differential polynomials in $z$ with coefficients in $\bar{F}$. Since $z$ is a zero of a linear differential polynomial, $V$ is a finite dimensional vector space. For any element $v \in V, \bar{F}\langle v\rangle \subseteq \bar{F}\langle z\rangle$ so that order of $v$ over $\bar{F}$ is $\leqq 1$. Let $A$ be the set of all elements $v$ in $V$ such that order of $v$ over $\overline{\bar{F}}$ is 1 . $A$ is not empty since $z \in A$. Of all the elements in $A$ choose $u$ such that the linear order of $u$ over $\bar{F}$ is least. We are going to show that the linear order of $u$ over $\bar{F}$ is 1 .

Let the linear order of $u$ over $\bar{F}$ be $n$ and let $W$ be the $n+1$ dimensional vector space over $\bar{F}$ of all linear differential polynomials in $u$ with coefficients in $\overline{\mathfrak{F}}$. For any $w \in W-\overline{\mathcal{F}}$ the following holds:

(1) $w \in A$ (i.e. $w$ is of order 1 over $\mathcal{F}$ ).

(2) Linear order of $w$ over $\bar{F}$ is $n$.

(3) If the $n$th order linear equation that $w$ satisfies over $\bar{F}$ is $M(y)=f, M(y)$ homogeneous, $f \in \overline{\mathcal{F}}$; then $M(y)$ is linearly irreducible.

To prove (1) note that $w \in V-\bar{F}$ and, since $\bar{F}$ is algebraically closed the order of $w$ over $\bar{F}$ is 1 . Since $w \in A$ the linear order of $w$ over $\bar{F}$ is $\geqq n$ (since $n$ was least) but $w \in W$ and each element in $W$ has linear order over $\overline{\mathcal{F}} \leqq n$. This proves (2). To prove (3) we note that if $M(y)$ $=N_{2}\left(N_{1}(y)\right), N_{1}(y)$ of positive order, then the linear order of $N_{1}(w)$ 
over $\bar{F}$ is the order of $N_{2}$ which is less than linear order of $w$ over $\bar{F}$ contradicting (2). Hence $M(y)$ is linearly irreducible over $\bar{F}$.

Now, by Theorem 2 , there exists $w \in W-\overline{\mathcal{F}}$ such that $w$ is a zero of a first order differential polynomial $Q(y) \in \bar{F}\{y\}$ the sum of whose highest degree terms is of order 1 . Since $\overline{\mathcal{F}}$ is algebraically closed the sum of the highest degree terms factors into linear factors with at least one of the factors $N_{1}(y)$ of order 1 . By Theorem 1 a generic zero of the prime differential ideal generated by $N_{1}(y)$ is a zero of $M(y)$ (since the generic zero is of order 1 over $\bar{F}$ ), so that $M(y)$ belongs to the prime differential ideal $\left\{N_{1}(y)\right\}$. Since $M(y)$ is linear (i.e. of the same degree as $N(y)), M(y)=N_{2}\left(N_{1}(y)\right)$. By (3) $M(y)$ is linearly irreducible so that $N_{2}(y)$ is of order zero and $M(y)$ is of order 1. By (2) this implies that $n=1$ and the linear order of $u$ over $\bar{F}$ is 1 . Since $u$ is of order 1 over $\mathcal{F}$ it follows that $z$ is algebraic over $\mathcal{F}\langle u\rangle$; this proves our theorem.

COROLlaRy. If a zero $z$ of a first order differential polynomial $P(y)$ $\in \mathcal{F}\{y\}$ is a zero of a linear differential polynomial $L(y) \in \mathcal{F}\{y\}$, then either $z$ is algebraic over $\mathfrak{F}$ or $P(y)$ is solvable by quadratures.

\section{REFERENCES}

1. E. R. Kolchin, Extension of differential fields, I, Ann. of Math. (2) vol. 43 (1942) pp. 724-729.

2. A. Loewy, Über die Irreduzibilität der linearen homogenen Substitusionsgruppen und Differentialgleichungen. Math. Ann. vol. 70 (1911) pp. 94-109.

3. H. Poincaré, Sur les groupes des equations lineaires, Acta Math. vol. 4 (1884) pp. 201-311.

4. J. F. Ritt, Differential algebra, New York, Amer. Math. Soc. Colloquium Publications, vol. 33, 1950.

5. L. Schlesinger, Handbuch der Theorie der linearen Differentialgleichungen, Leipzig, 1897.

Stevens Institute of Technology 